\title{
Study on the Radial Vibration of a Thin Annular Vibrator with Multi-Radial Slots
}

\author{
Liu Shiqing, ${ }^{1,}$, Ma Leilei ${ }^{1}$, Liu Yifeng ${ }^{2}$, Li Dan $^{1}$, Chen Zhaojiang ${ }^{1}$ \\ ${ }^{1}$ College of Mathematics, Physics and Information Engineering, Zhejiang Normal University, Jinhua, China \\ ${ }^{2}$ Institute of Acoustics, Chinese Academy of Sciences, Beijing, China
}

\section{Email address:}

zjnulsq@163.com (Liu Shiqing),981924025@qq.com (Ma Leilei)

${ }^{*}$ Corresponding author

\section{To cite this article:}

Liu Shiqing, Ma Leilei, Liu Yifeng, Li Dan, Chen Zhaojiang. Study on the Radial Vibration of a Thin Annular Vibrator with Multi-Radial Slots. American Journal of Applied Scientific Research. Vol. 5, No. 1, 2019, pp. 1-5. doi: 10.11648/j.ajasr.20190501.11

Received: December 31, 2018; Accepted: January 21, 2019; Published: March 28, 2019

\begin{abstract}
The radial vibration of a thin annular vibrator with multiple radial slots is studied. Based on the electro-mechanical analogy, the equivalent circuit and the frequency equation of the slotting thin annular vibrator in radial vibration are derived. By numerical simulations, the influences of the radius ratio of the thin annular vibrator on its displacement amplitude amplification factor and the first- and the second-order resonance frequency have been investigated. The analysis results manifest that the first-order amplitude amplification factor and the resonance frequency of the ring increase with the increases of the radius ratio, and the situation is the opposite for the relation between the second-order amplitude amplification factor and the radius ratio. The Finite Element Method (FEM) is employed for the simulation of the radial vibration of the thin annular vibrator. The FEM results are in good agreement with the analytical solution.
\end{abstract}

Keywords: Thin Annular Vibrator, Multiple Radial Slots, Radial Vibration, Resonance Frequency, The Amplitude Amplification Factor, FEM Simulation

\section{Introduction}

In the field of high power ultrasonic, vibrators can be designed in various shapes and excited to vibrate in different modes for some particular applications [1-2]. By making some diagonal slots on a concentrator rod (or horns), the longitudinal vibration of the rod could be converted into elliptical motion at the slits part of the rod, which can be used for ultrasonic motor driver stator and ultrasonic elliptical vibration cutting [3-4]. In the application of ultrasonic welding, large cross-sectional rods are usually used as tool head for the transfer of high intensity ultrasound. The transverse size of the rod is always at large close to or more than one-half of a wavelength. Therefore, the strong coupled vibration will exist between the transverse and longitudinal directions and then led to great decrease of the energy transfer efficiency of the tool. In order to reduce the coupled vibrations in transverse and longitudinal direction, it is usually to cut a series of parallel slits along the vibration direction of the large cross-sectional size rod, which can significantly improve the amplitude and transfer efficiency of the ultrasonic energy [5]. In recent years, some kinds of radial vibration ultrasonic concentrator have been developed for ultrasonic manufacturing processes and wire drawing [6-7]. By using Ritz method, the Three Dimension vibration performance of the circular ring vibrators with nonlinear variable thickness section of taper, exponent and step shape were studied, and the eigenvalues of the frequency equation and mode shapes of them were obtained [8]. Liu \& Lin proposed a ring vibrator and studied. Based on the electro-mechanical analogy, the equivalent circuit and the frequency equation of the vibrator in radial vibration were obtained [9-10]. Beside, Lin and $\mathrm{Xu}$ proposed another king of hollow cylinder vibrator with large geometrical dimensions, the coupled vibration of were studied and the resonance frequency equation of the vibrator in coupled vibration were derived [11].

In order to further improve the performance of the disc or circular ring ultrasonic vibrator, an annular concentrator with multiple slots along the radial direction is proposed and studied. Based on the elasticity mechanics and electro-mechanical analogy, the equivalent circuit and 
frequency equation of the concentrator in radial vibration are derived, the relationship between the frequency, the anti-frequency in radial vibration and the geometrical dimensions of the annular concentrator are studied and simulated by using FEM (Finite Element Method).

\section{Analysis on the Radial Vibration of a Slot-Type Annular Vibrator}

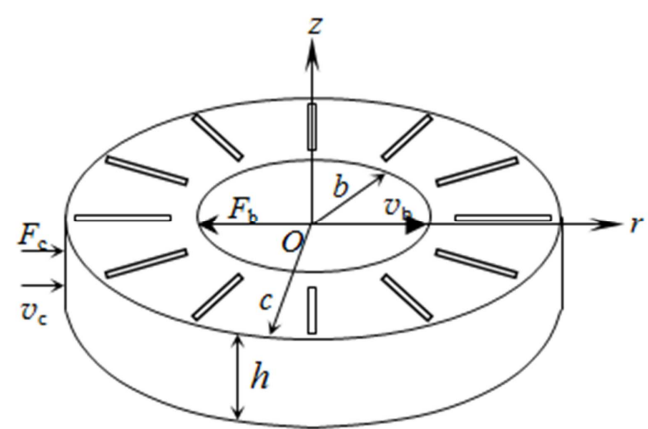

Figure 1. The thin annular vibrator with radial slots.

A thin annular elastic vibrator with multiple slots in its radial direction is shown in Figure 1. $b$ and $c$ are the inner and outer radius of the vibrator, respectively. And its thickness is $\mathrm{h}$ $F_{\mathrm{b}}, F_{\mathrm{c}}$ and $v_{\mathrm{b}}, v_{\mathrm{c}}$ are the external forces and velocities at the inner and outer radiating surfaces of the annular vibrator, respectively. In the cylindrical coordinate system, the motion equation of the vibrator in radial vibration is expressed as following [9]:

$$
\rho \frac{\partial^{2} u_{\mathrm{r}}}{\partial t^{2}}=\frac{\partial T_{\mathrm{r}}}{\partial r}+\frac{1}{r} \cdot \frac{\partial T_{\mathrm{r} \theta}}{\partial \theta}+\frac{\partial T_{\mathrm{rz}}}{\partial z}+\frac{T_{\mathrm{r}}-T_{\theta}}{r}
$$

Here, $u_{\mathrm{r}}$ is the radial vibration displacement. $\mathrm{T}_{r}, \mathrm{~T}_{\theta}, \mathrm{T}_{r \theta}$ and $\mathrm{T}_{r z}$ are the respective components of the internal stress, respectively. In polar coordinates, the relationship between strain and displacement can be expressed as following:

$$
S_{\mathrm{r}}=\frac{\partial u_{\mathrm{r}}}{\partial r}
$$

Here, $S_{\mathrm{r}}$ is the vibrator's strain component. Known by the general Hooke's law, the relationship between stress and strain can be written as following:

$$
S_{\mathrm{r}}=\frac{1}{E}\left[T_{\mathrm{r}}-v\left(T_{\theta}+T_{\mathrm{z}}\right)\right]
$$

Here, $E$ and $v$ are the elastic modulus and the Poisson coefficient of the material, respectively.

The vibrator are actually performs complex three-dimensional coupled vibration. We assume that the thickness of the radially slotted annular vibrator is much smaller than its diameter. And if the number and length of the slots are enough large to make the lateral size of each interval between two adjacent slots far less than its length, and less than a quarter of a wavelength. Then due to the segmentation of the slots, the shear stress in disc can be approximately considered to be zero, and the vibration can be approximately regarded as a longitudinal vibration, which helps improve the radial displacement amplitude ratio and energy transfer efficiency of the slot-type annular vibrator.

From the above assumptions, the radial stress $T_{\mathrm{r}}$ is not equal to zero; the rest of the stress components and $v$ are approximately considered to be zero. The radial wave equation of the vibrator can be simplify by the above equations as:

$$
\frac{\partial^{2} u_{\mathrm{r}}}{\partial r^{2}}+\frac{1}{r} \cdot \frac{\partial u_{\mathrm{r}}}{\partial r}=\frac{1}{v_{\mathrm{r}}^{2}} \cdot \frac{\partial^{2} u_{\mathrm{r}}}{\partial t^{2}}
$$

Here, $v_{r}=\sqrt{E / \rho}$ is the radial vibration wave velocity of the vibrator. The general solution to Equation (4) can be obtained as following:

$$
u_{\mathrm{r}}(r, t)=\left[C_{1} J_{0}(k r)+C_{2} Y_{0}(k r)\right] \cdot \exp (\mathrm{j} \omega \mathrm{t})
$$

The radial velocity of the vibrator can be obtained from Equation (5), as:

$$
v_{\mathrm{r}}=\mathrm{j} \omega\left[C_{1} J_{0}(k r)+C_{2} Y_{0}(k r)\right] \cdot \exp (\mathrm{j} \omega \mathrm{t})
$$

The radial stress in the vibrator can be written as:

$$
T_{\mathrm{r}}=-E k\left[C_{1} J_{1}(k r)+C_{2} Y_{1}(k r)\right] \cdot \exp (\mathrm{j} \omega \mathrm{t})
$$

Here, $k=\omega^{\prime} v_{\mathrm{r}}$ is the wavenumber and the angular frequency. $J_{0}(k r)$ and $Y_{0}(k r)$ are the first and second kinds of zero-order Bessel functions, respectively. $C_{1}$ and $C_{2}$ are two undetermined constants determined by the radial vibration boundary conditions of the vibrator.

\subsection{The Amplitude Amplification Factor of the Thin Annular Vibrator with Radial Slots}

Let the amplitude at the outer surface of the vibrator be $u_{c}$. For free radial vibration, the boundary condition of the surface is expressed as following:

$$
\left.u_{\mathrm{r}}(r)\right|_{\mathrm{r}=\mathrm{c}}=u_{c} ;\left.T_{\mathrm{r}}(r)\right|_{\mathrm{r}=\mathrm{b}}==0
$$

Substituting Equations (5) and (7) into Equation (8), yields:

$$
\begin{aligned}
& C_{1} J_{0}(k c)+C_{2} Y_{0}(k c)=u_{c} \\
& C_{1} J_{1}(k b)+C_{2} Y_{1}(k b)=0
\end{aligned}
$$

The radial displacement distribution function of the vibrator is given by Equations (9) and (10), as:

$$
u_{r}(r)=\frac{J_{1}(k b) Y_{0}(k r)-Y_{1}(k b) J_{0}(k r)}{J_{1}(k b) Y_{0}(k c)-J_{0}(k c) Y_{1}(k b)} u_{c}
$$

Here, the annular vibrator's amplitude amplification factor is defined as the ratio of the internal and external surface amplitudes, which can be expressed as following: 


$$
M=\frac{u_{b}}{u_{c}}=\frac{J_{1}(k b) Y_{0}(k b)-Y_{1}(k b) J_{0}(k b)}{J_{1}(k b) Y_{0}(k c)-J_{0}(k c) Y_{1}(k b)}
$$

\subsection{The Equivalent Circuit of the Thin Annular Vibrator with Radial Slots}

From Figure 1, when $\left.v_{\mathrm{r}}\right|_{\mathrm{r}=\mathrm{c}}=-v_{\mathrm{c}}$ and $\left.v_{\mathrm{r}}\right|_{\mathrm{r}=\mathrm{b}}=v_{\mathrm{b}}$, yields:

$$
\begin{aligned}
& C_{1}=\frac{1}{\mathrm{j} \omega} \cdot \frac{\left[Y_{0}(k b) v_{\mathrm{a}}+Y_{0}(k c) v_{\mathrm{b}}\right] \exp (-\mathrm{j} \omega t)}{J_{0}(k b) Y_{0}(k c)-J_{0}(k c) Y_{0}(k b)} \\
& C_{2}=\frac{-1}{\mathrm{j} \omega} \cdot \frac{\left[J_{0}(k b) v_{\mathrm{c}}+J_{0}(k a) v_{\mathrm{b}}\right] \exp (-\mathrm{j} \omega t)}{J_{0}(k b) Y_{0}(k c)-J_{0}(k c) Y_{0}(k b)}
\end{aligned}
$$

In the Figure 1, we have $\left.F_{\mathrm{r}}\right|_{\mathrm{r}=\mathrm{c}}=\left.T_{\mathrm{r}}\right|_{\mathrm{r}=\mathrm{c}} \cdot S_{\mathrm{c}}=-F_{\mathrm{c}}$, $\left.F_{\mathrm{r}}\right|_{\mathrm{r}=\mathrm{b}}=\left.T_{\mathrm{r}}\right|_{\mathrm{r}=\mathrm{b}} \cdot S_{\mathrm{b}}=-F_{\mathrm{b}}$. Substituting Equations (13) and (14) into Equation (7), yields:

$$
\begin{aligned}
F_{\mathrm{c}}= & \frac{-k E S_{\mathrm{c}}}{\mathrm{j} \omega}\left[\frac{J_{0}(k b) Y_{1}(k c)-J_{1}(k c) Y_{0}(k b)}{J_{0}(k b) Y_{0}(k c)-J_{0}(k c) Y_{0}(k b)} v_{\mathrm{c}}\right)+ \\
& \left.\frac{J_{0}(k c) Y_{1}(k c)-J_{1}(k c) Y_{0}(k c)}{J_{0}(k b) Y_{0}(k c)-J_{0}(k c) Y_{0}(k b)} v_{\mathrm{b}}\right] \\
F_{\mathrm{b}}= & \frac{-k E S_{\mathrm{b}}}{\mathrm{j} \omega}\left[\frac{J_{0}(k b) Y_{1}(k b)-J_{1}(k b) Y_{0}(k b)}{J_{0}(k b) Y_{0}(k c)-J_{0}(k c) Y_{0}(k b)} v_{\mathrm{c}}+\right. \\
& \left.\frac{J_{0}(k c) Y_{1}(k b)-J_{1}(k b) Y_{0}(k c)}{J_{0}(k b) Y_{0}(k c)-J_{0}(k c) Y_{0}(k b)} v_{\mathrm{b}}\right]
\end{aligned}
$$

Here, $S_{\mathrm{b}}=2 \pi b h, S_{\mathrm{c}}=2 \pi \mathrm{ch}$ are the inner and outer areas of the vibrator, respectively. Equations (15) and (16) can be further transformed as the following forms:

$$
\begin{aligned}
& F_{\mathrm{c}}=\left(z_{2}+z_{3}\right) v_{\mathrm{c}}+z_{3} v_{\mathrm{b}} \\
& F_{\mathrm{b}}=z_{3} v_{\mathrm{c}}+\left(z_{1}+z_{3}\right) v_{\mathrm{b}}
\end{aligned}
$$

Here, three impedances can be expressed as following:

$$
\begin{gathered}
z_{1}=\mathrm{j} z_{\mathrm{c}} \frac{J_{0}(k b) Y_{1}(k c)-J_{1}(k c) Y_{0}(k b)}{J_{0}(k b) Y_{0}(k c)-J_{0}(k c) Y_{0}(k b)}-z_{3} \\
z_{2}=\mathrm{j} z_{\mathrm{b}} \frac{J_{0}(k c) Y_{1}(k b)-J_{1}(k b) Y_{0}(k c)}{J_{0}(k b) Y_{0}(k c)-J_{0}(k c) Y_{0}(k b)}-z_{3} \\
z_{3}=\frac{\mathrm{j}}{\omega} \cdot \frac{4 E h}{J_{0}(k c) Y_{0}(k b)-J_{0}(k b) Y_{0}(k c)}
\end{gathered}
$$

Here, $z_{\mathrm{b}}=\rho v_{\mathrm{r}} S_{\mathrm{b}}, z_{\mathrm{c}}=\rho v_{\mathrm{r}} S_{\mathrm{c}}$.

In summary, the electro-mechanical equivalent circuit of radially slotted annular vibrator as shown in Figure 2 can be obtained by the equivalent circuit theory.

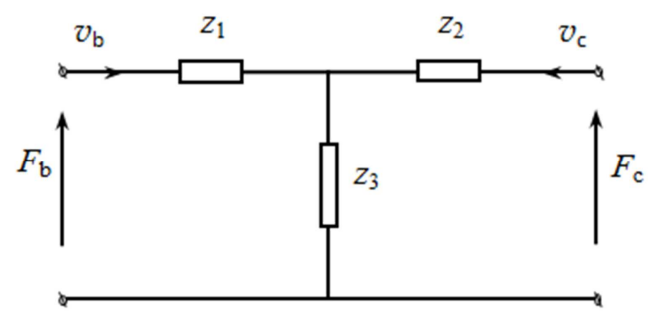

Figure 2. The equivalent circuit of the annular vibrator with radial slots.

When there are no external forces acting on the inner and outer surfaces of the annular vibrator, from Figure 2, the frequency equation of the vibrator can be obtained as the following forms:

$$
\operatorname{Im}\left(\frac{z_{1} z_{2}+z_{2} z_{3}+z_{1} z_{3}}{z_{2}+z_{3}}\right)=0
$$

Substituting the expressions of $z_{1}, z_{2}, z_{3}$ into Equation (22), the concrete expression of the resonant frequency equation can be derived as follow:

$$
\left|\begin{array}{ll}
J_{1}(k c) & J_{0}(k b) \\
Y_{1}(k c) & Y_{0}(k b)
\end{array}\right| \cdot\left|\begin{array}{ll}
J_{1}(k b) & J_{0}(k c) \\
Y_{1}(k b) & Y_{0}(k c)
\end{array}\right|=\frac{16 E^{2} h^{2}}{\omega^{2} z_{c} z_{b}}
$$

The resonant frequency of a radially slotted annular vibrator dependence with geometrical dimensions can be obtained by numerical calculation.

\section{Numerical Calculations and Finite Element Simulation}

\subsection{The Relationship Between the Resonance Frequency and the Amplitude Amplification Factor of the Vibrator with Its Radius Ratio}

In the calculations, as an example, the standard material parameters of aluminum is listed as followings, $\rho=2700 \mathrm{~kg} / \mathrm{m}^{3}$, $\nu=0.34$ and $E=71.5 \mathrm{GPa}$. The geometric parameters of $c$ and $h$ are $64 \mathrm{~mm}$ and $7 \mathrm{~mm}$, respectively. A parameter $\gamma_{\text {of inner and }}$ outer radius ratio is introduced and defined as $\gamma=b / c$. In the calculation, keep all the above parameters to be constant, changing the inner diameter $b$ of the ring for changing its radius ratio. The relationship between the resonance frequency $f_{r}$ and the amplitude amplification factor $M$ with the radius ratio of the radially slotted annular vibrator is shown in Figure 3 and Figure 4, respectively.

Figure 3 shows that the first and second resonance frequencies of the vibrator are increase monotonously with the increases of the radius ratio, which are different from the radial vibration characteristics of the non-slotted annular vibrator [9]. 


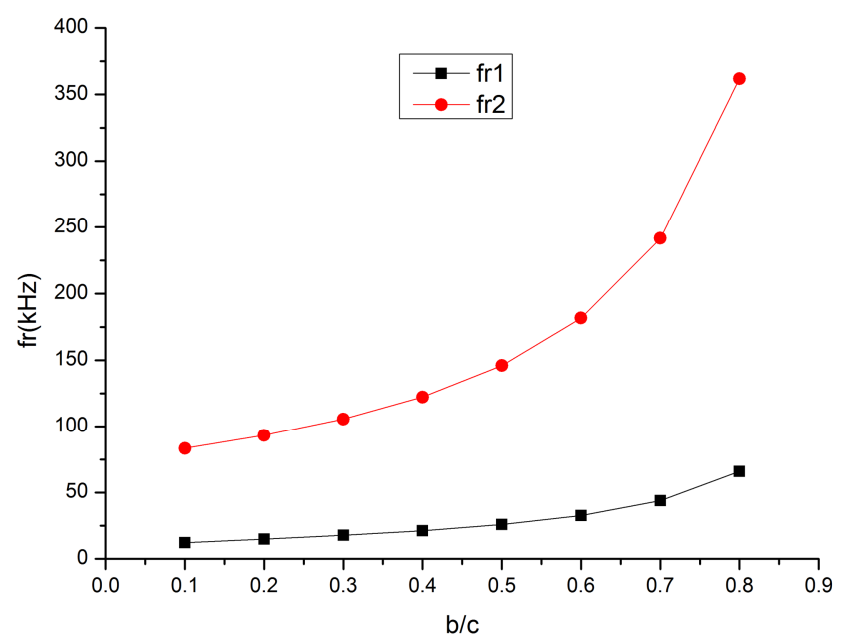

Figure 3. The relationship between the first and second resonance frequencies of the vibrator and radius ratio.

From Figure 4, It can be seen that the first-order amplitude amplification factor of the vibrator increases with the radius ratio increases (decreases in the ring width), but it is not significant; The amplitude amplification factor of the second-order decreases monotonically with the radius ratio increases, and it is much larger than the first-order amplitude amplification factor at a relatively small radius.

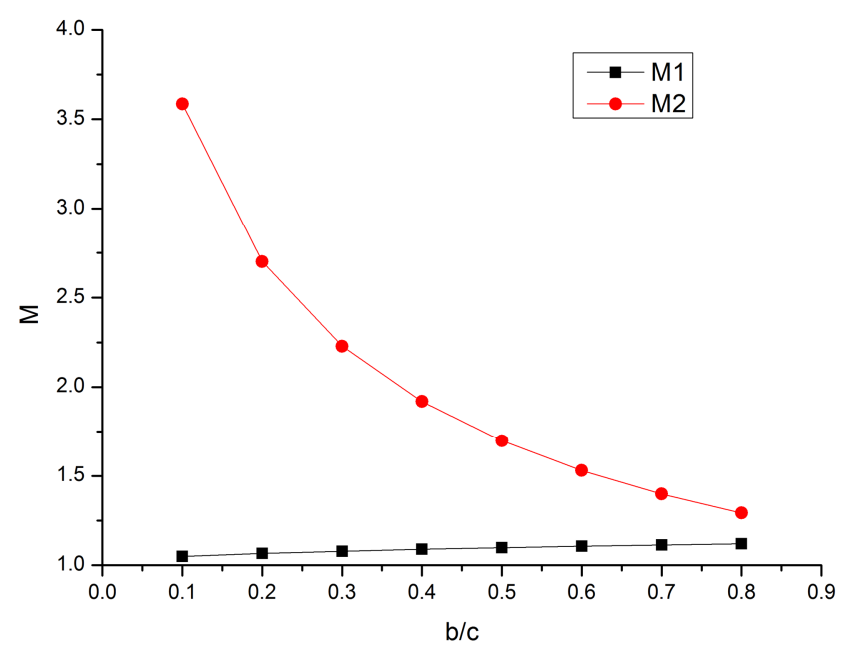

Figure 4. The relationship between the first and second amplitude amplification factor of the vibrator and radius ratio.

\subsection{The Relationship Between the Resonance Frequency Relative Error with the Number of Slots}

The finite element software COMSOL Multiphysics are used to simulate the radial vibration modes of the vibrator. The radially slotted annular vibrator material is aluminum, and material parameters are the same as above. The geometric parameters of $c, b$ and $h$ are $32 \mathrm{~mm}, 15 \mathrm{~mm}$ and 7 $\mathrm{mm}$, respectively. And slots length are $11.5 \mathrm{~mm}$. The numerical simulation is performed for the five cases, which the number of slots being 12, 15, 18, 21 and 24, respectively. The theoretical and simulated fundamental resonance frequencies of the vibrator is shown in Table 1, where $f_{n}$ and $f_{t}$ are the simulated and the theoretical result, respectively; the relative error is defined as $\left|f_{n}-f_{t}\right| / f_{n}$. As can be seen from Table 1, when there are lack of the slots, the deviation between theoretical and simulated results is large, but the relative error decreases gradually as the number of slots increases in the vibrator. This is because the theory is derived based on the assumption that the number of slots is sufficient.

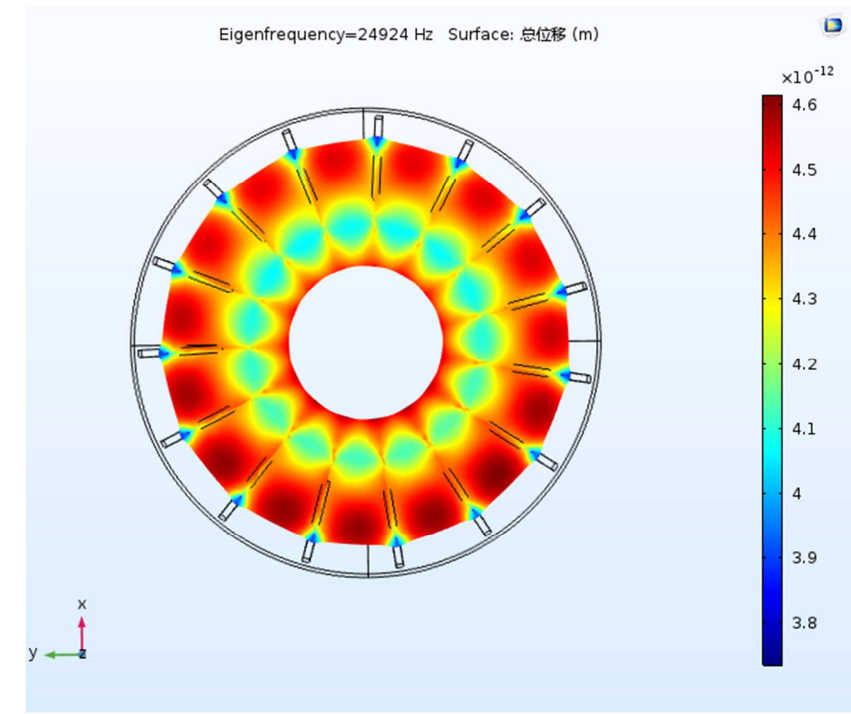

Figure 5. The radial first-order resonant mode of the annular vibrator with 15 slots.

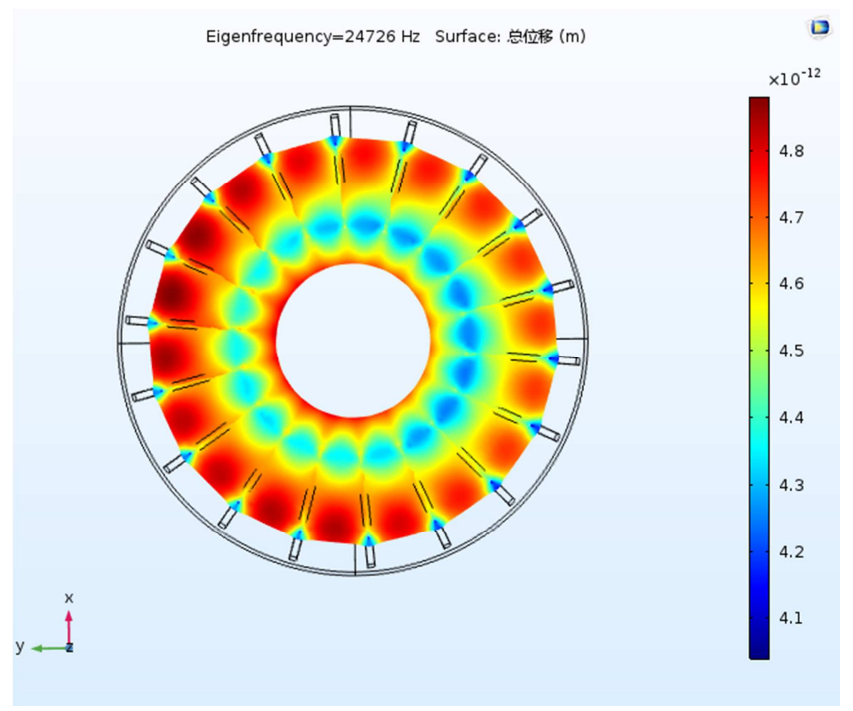

Figure 6. The radial first-order resonant mode of the annular vibrator with 18 slots.

Table 1. The theoretical and simulated fundamental resonance frequencies of the annular vibrator.

\begin{tabular}{llll}
\hline \multicolumn{4}{l}{ Theoretical value Simulation error } \\
\hline $\mathbf{N}$ & $\left(\boldsymbol{f}_{\boldsymbol{t}} / \mathbf{H z}\right)$ & $\left(\boldsymbol{f}_{\boldsymbol{n}} / \mathbf{H z}\right)$ & $(\mathbf{\%})$ \\
\hline 12 & 24251 & 25338 & 4.29 \\
15 & 24251 & 24924 & 2.70 \\
18 & 24251 & 24726 & 1.92 \\
21 & 24251 & 24655 & 1.64 \\
24 & 24251 & 24653 & 1.63 \\
\hline
\end{tabular}


Figure 5 and Figure 6 are the vibrator's fundamental resonance mode shapes and corresponding displacement vector distribution with the number of slots 15, 18, respectively. In the latest study with a slotted vibrator, the vibrator is divided into three regions and studied separately [8]. Similarly, the relationship between the first and second resonance frequencies and the amplitude amplification factor of the vibrator with the number, depth, and width of the slot are obtained. This will be elaborated in another article. In addition, within the allowable error range, a larger displacement amplitude ratio and higher energy transfer efficiency can be obtained as long as the slot size is properly selected in practical applications.

\section{Conclusions}

(1) In this paper, the radial vibration characteristics of a radially slotted thin annular vibrator are studied, and its electro-mechanical equivalent circuit and resonance frequency equation are derived. The relationship between the resonance frequency and the amplitude amplification factor of the vibrator with its geometric dimension is discussed by using numerical calculations.

(2) The research above shows that the first and second resonance frequencies of the radially slotted annular vibrator increase monotonously with the radius ratio increases. Besides, the first-order amplitude amplification factor of the vibrator increases with its radius ratio increases, while the second-order frequency decreases monotonously. And the second-order amplitude amplification factor is much larger than that of the first-order while the radius is relatively small, that is, the ring width is large.

(3) It is evident that the amplitude amplification factor of the vibrator is not only depend on the selected material but also the number of slots. For engineering application, the performance of the vibrator at the second-order resonant is better than the first one. On the one hand, the second-order resonance mode can get a larger displacement amplitude ratio and higher energy transfer efficiency. On the other hand, there is a displacement node circle line in the thin annular vibrator at the second radial resonance, which is convenient to fix the vibrator.

\section{Acknowledgements}

The authors wish to acknowledge to The National Natural Science Foundation of China for its financial support (Project Number: 11874326, 11874327).

\section{References}

[1] LIN Shuyu. Study on the longitudinal torsional composite mode exponential ultrasonic horns [J]. Ultrasonics, 1996, 34(7): 757-762.

[2] N A Shvetsova, D I Makarev, I A Shvetsov, et al. New Techniques and Designs of Focusing Piezoelectric Transducers for Ultrasonic Diagnostics and Therapy [J]. Bulletin of the Russian Academy of Sciences: Physics, 2018, 82 (3): 251-256.

[3] PI Jun. Longitudinal torsional vibration conversion of ring chute transmission rod $[\mathrm{J}]$. Chinese Journal of Mechanical Engineering, 2008, 44 (5): 242-248.

[4] J Tsujino, $\mathrm{T}$ ueoka, $\mathrm{K}$ Otoda, et al. One-dimensional longitudinal torsional vibration converter with multiple diagonally slitted parts [J]. Ultrasonics, 2000, 38 (8): 72-76.

[5] LIN Dzon-mou. Equivalent circuit of a vibrating system magnetostrictive transducer-concentrator-sample. Procesdings of vibration problems (Poland), 1963, 4: 175-184.

[6] K Siegert, J Uner. Superimposing ultrasonic waves on the dies in tube and wire drawing [J]. Journal of Engineering Material and Technology, 2001, 123 (4): 517-523.

[7] D H Xiang, X T Zhi, G X Yue, B Zhao, Q T Fan. Experimental Study on Ultrasonic Vibration Dressing Wheel [J]. Advanced Materials Research, 2010, 135: 260-264.

[8] KANG Jae-Hoon. Three-dimensional vibration analysis of thick, circular and annular plates with nonlinear thickness variation [J]. Computers and Structures, 2003, 81: 1663-1675.

[9] LIU Shiqing, YANG Xianli, LI Luhua. Radial vibration of a slit-type annular ultrasonic concentrator [J]. Chinese Journal of Acoustics, 2015, 34 (2): 153-165.

[10] LIN Shuyu. Radial vibration and equivalent circuit of thin elastic circular rings [J]. ACTA ACUSTICA, 2003, 28 (2): 102-106.

[11] LIN Shuyu. Coupled vibration of isotropic metal hollow cylinders with lager geometrical dimensions [J]. Journal of Sound and Vibration, 2007, 305 (1-2): 308-316. 